Published in final edited form as:

J Am Chem Soc. 2016 October 5; 138(39): 12739-12742. doi:10.1021/jacs.6b07647.

\title{
Blue-Emitting Arylalkynyl Naphthalene Derivatives via a Hexadehydro-Diels-Alder Cascade Reaction
}

\author{
Feng $\mathrm{Xu}^{\dagger}$, Kyle W. Hershey ${ }^{\ddagger}$, Russell J. Holmes ${ }^{\ddagger}$, and Thomas R. Hoye ${ }^{\dagger,}{ }^{,}$ \\ tDepartment of Chemistry, University of Minnesota, Minneapolis, Minnesota 55455, United States \\ ‡Department of Chemical Engineering and Materials Science, University of Minnesota, \\ Minneapolis, Minnesota 55455, United States
}

\begin{abstract}
We describe here three alkynyl substituted naphthalenes that display promising luminescence characteristics. Each compound is easily and efficiently synthesized in three steps by capitalizing on the hexadehydro-Diels-Alder (HDDA) cycloisomerization reaction in which an intermediate benzyne is captured by tetraphenylcyclopentadienone, a classical trap for benzyne itself. These compounds luminesce in the deep blue when stimulated either optically (i.e., photoluminescence in both solution and solid films) or electrically [in a light-emitting diode (LED)]. The photophysical properties are relatively insensitive to the electronic nature of the substituents $(\mathrm{H}$, $\mathrm{OMe}, \mathrm{CO}_{2} \mathrm{Me}$ ) that define these otherwise identical compounds. Overall, our observations suggest that the twisted nature of the five adjacent aryl groups serves to minimize the intermolecular interaction between core naphthalene units in different sample morphologies. These compounds represent promising leads for the identification of others of value as the emissive component of organic LEDs (OLEDs).
\end{abstract}

It is known that $o$-benzyne (or 1,2-dehydrobenzene, 2) is the parent member of one of the most versatile classes of reactive intermediates in all of chemistry. Among the myriad modes of reaction into which benzynes enter is the Diels-Alder (DA) [4+2] cycloaddition reaction. The strained alkyne within the benzyne (the $2 \pi$ component) engages a wide variety of 1,3 dienes (the $4 \pi$ component). Incorporation of structural complexity into the benzyne moiety using conventional methods of benzyne generation ${ }^{1}$ becomes increasingly challenging because these all involve producing the benzyne by removal of two adjacent atoms or groups from an aromatic precursor (1 to 2, Figure 1a). In contrast the hexadehydro-Diels-Alder (HDDA) reaction, ${ }^{2}$ which capitalizes on the simple thermal cycloisomerization of a $1,3,-$ diyne with a tethered alkyne diynophile (5 to $\mathbf{6}$, Figure $1 \mathrm{~b}$ ), ${ }^{3}$ results in de novo construction

\footnotetext{
*Corresponding Author. hoye@umn.edu.

ASSOCIATED CONTENT

Supporting Information

The Supporting Information is available free of charge on the ACS Publications website at DOI: 10.1021/jacs.6b07647.

Details for preparation of new compounds (PDF)

Data for $\mathrm{C}_{57} \mathrm{H}_{44} \mathrm{O}_{6}(\mathrm{CIF})$
}

The authors declare the following competing financial interest(s): FX and TRH are inventors on a provisional U.S. patent application. $\mathrm{RJH}$ is a member of The Dow Chemical Company Technical Advisory Board. 
of the six-membered, carbocyclic benzyne moiety. The HDDA reaction is highly amenable to the incorporation of substituents and structural variants (A-C, Y, and Z in Figure 1b) into the precursors, intermediate benzynes, and trapped products. ${ }^{4}$ The HDDA reaction readily accommodates conjugated, $\pi$-bond-rich linker units and substituents.

With an eye toward the rapid synthesis of compounds having new structural motifs, novel chromophores, and potentially valuable photophysical properties, we have begun exploring HDDA cascades [i.e., the sequential (and rate determining) generation followed by rapid ${ }^{5}$ in situ trapping of an HDDA-generated benzyne] in which the trapping step is an independent, second DA reaction. For example, we envision that trapping of $\mathbf{6}$ by the diene unit (blue) in one of a multitude of trapping agents having the generalized structure 7 will lead to many diverse, chromophoric, polycyclic derivatives like $\mathbf{8}$.

Two facts guided the thinking that initiated the studies whose results we report here. (i) The DA reaction of $o$-benzyne (2) with tetraphenylcyclopentadienone (3, TPCP) efficiently produces 1,2,3,4-tetraphenylnaphthalene (4), via thermal extrusion of $\mathrm{CO}$ from the initial norbornenone DA adduct. ${ }^{6}$ (ii) Alkynyl substituents on polycyclic aromatic rings often impart interesting or valuable excited state emission properties. ${ }^{7}$ Accordingly, one of the early reactions we explored was that between the tetrayne $9 \mathbf{a}$ and TPCP $(\mathbf{3}$, Figure 2$){ }^{8}$ The symmetry of this HDDA substrate make it attractive from a preparative perspective. It is made in two simple steps. ${ }^{9}$ This HDDA cascade, using 1.5 equiv of $\mathbf{3}$, proceeded cleanly at $80{ }^{\circ} \mathrm{C}$ over the course of $12 \mathrm{~h}$ and produced a near quantitative yield ( $82 \%$ following three recrystallizations) of the naphthalene adduct 10a. An added advantage of using a tetrayne substrate is that the intermediate benzyne, here $\mathbf{1 1}$, and, consequently, the naphthalene formed following $\mathrm{CO}$ extrusion from 12, bears an arylethynyl substituent. The product 10a showed, qualitatively, strong emissive properties that were blue to the eye. This was true for the material whether in a solid film, adsorbed on silica gel (tlc analysis), or dissolved in solution.

In view of these promising properties, we decided to probe the effect of the electronic character of the aryl substituents. Hence, we synthesized two additional analogs in which the phenyl ring was modified by the presence of a pair of electron-donating (10b) as well as electron-withdrawing (10c) groups. These analogs were readily prepared by an entirely parallel and equally efficient sequence ${ }^{9}$ of reactions.

Figure 3a shows the solution absorption $\left(10^{-5} \mathrm{M}\right)$ and photoluminescence (PL, $\left.10^{-6} \mathrm{M}\right)$ spectra of 10a-10c in tetrahydrofuran solution. The PL quantum yields for each compound are $11.0 \pm 2.6 \%, 4.8 \pm 1.3 \%$, and $56 \pm 8 \%$, for $10 \mathrm{a}, 10 \mathrm{~b}$, and $10 \mathrm{c}$, respectively. There was nearly no change ( $\mathcal{2} \mathrm{nm}$ ) in the $\lambda_{\max }$ of emission for 10a and 10b; a red shift of $10 \mathrm{~nm}$ in that $\lambda_{\max }$ was seen for 10c.

Surprisingly, these compounds also showed a behavior typical of aggregation-induced emission (AIE).$^{10}$ Namely, as the strong solvent THF was exchanged for increasing amounts of the nonsolvent water, the efficiency of blue emission grew (see SI). As an example, compare the left and middle vials in Figure 3b, which contain the same amount of $\mathbf{1 0 b}$ in pure THF vs $10 \%$ THF in water, respectively. However, given the array of five orthogonal 
aryl substituents, one would not expect that two (or more) molecules of $\mathbf{1 0}$ would be able to reside close enough to show substantial excimeric behavior. Perhaps the planar (arylethynyl)-naphthalene portion of the molecule provides enough of a footprint for close association of molecular orbitals from two molecules. We do note that a topologically related compound, 1-methyl-1,2,3,4,5-pentaphenylsilole, also shows significantly enhanced emission when placed under aggregate-inducing conditions. ${ }^{11}$

Given the promising solution PL efficiencies as well as the qualitative emission seen from solid-state samples (third vial in Figure 3b), the emissive properties of all three compounds were investigated in thin films to explore their possible utility as emissive species in blue organic light-emitting diodes (OLEDs). OLEDs are attractive for both display and white lighting applications due to their (i) tunable spectral characteristics, (ii) relatively high luminescence efficiency, and (iii) compatibility with high throughput, large-area processing. ${ }^{12}$ The development of novel light-emitting molecules for efficient, deep-blue fluorescence remains an important area of research for the realization of high performance, low-cost OLED-based displays. ${ }^{13}$

To probe thin film PL, each compound was examined both as a pure film (100\%) as well as in a host-guest arrangement with the wide energy gap material 1,4bis(triphenylsilyl)benzene (UGH2) serving as host. Thin films were deposited on cleaned quartz substrates by high vacuum thermal evaporation $\left(<10^{-7}\right.$ Torr). It is notable that following deposition of each of the naphthalenes $10 \mathbf{a}-\mathbf{c}$, there was virtually no residue remaining in the heating crucible, suggesting little if any thermal degradation of these compounds. In fact, this point could be preliminarily established for samples of $\mathbf{1 0 a}-\mathbf{c}$ by observing (i) their sublimation in common laboratory apparatus with essentially no decomposition and (ii) their robust nature when held open in the air at a temperature of $2300{ }^{\circ} \mathrm{C}$, where they showed slight colorization but no sign of any significant decomposition. Finally, examination by differential scanning calorimetry again gave no indication of decomposition (exotherm) below $30{ }^{\circ} \mathrm{C}$ (for 10a and 10b) or $310^{\circ} \mathrm{C}$ (for 10c), and thermogravimetric analysis showed mass loss of greater than $5 \%$ only at temperatures $>336-357^{\circ} \mathrm{C}$ (see SI).

Films composed of host and guest (UGH2 and 10) in the ratios of 96:4, 80:20, and 0:100 (i.e., 4\%, 20\%, and 100\% loading, respectively) were studied. The thin film PL spectra of the $20 \%$ samples of each of 10a-c are shown in Figure $4 \mathrm{a}$. The solution and film emission maxima (Figures 3a vs $4 \mathrm{a}$ ) are similar, suggesting that there may not be intimate contact in the film samples. The twisted nature of the five aryl substituents on the central naphthalene ring as well as the quasi-orthogonal orientation of the pair of malonate carbomethoxy groups may be responsible for preventing close association between molecules of $\mathbf{1 0}$ in films. These features can be seen in the single crystal X-ray crystallographic structure shown in Figure 4b. The PL emission spectra of all three compounds showed a red shift of between 10 and 20 $\mathrm{nm}$ across the extreme concentrations of $4 \%$ vs $100 \%$ content of $\mathbf{1 0}$. This could be a result of at least some degree of aggregation with increasing concentration in the film, a response to a change in bulk dielectric properties or to differing intermolecular restraints that change the geometry of the excited states. DFT calculations (M06-2X/6-31G(d)) of the optimized 
lowest energy conformer for each of 10a-c showed both the twisted the ethynyl linker, quite similar to those features seen for the single crystal (see SI).

OLEDs using each of 10a-c as the emissive layer were constructed by high vacuum thermal evaporation. Devices (Figures 5a-c) were deposited on glass substrates, prepatterned with a semitransparent, hole-injecting anode of indium-tinoxide. These substrates were initially spin-coated with a $40 \mathrm{~nm}$-thick planarizing layer of the ionomeric mixture of poly $(3,4-$ ethylenedioxythiophene) and polystyrenesulfonate (PE-DOT:PSS) prior to vacuum deposition. The device architecture consisted of a $30 \mathrm{~nm}$-thick hole transport layer (HTL) of $4,4^{\prime}$-cyclohexylidenebis[ $N, N$-bis(4-methylphenyl)benzenamine] (TAPC), a $20 \mathrm{~nm}$-thick emissive layer of 10a, 10b, or 10c doped into the wide energy gap host UGH2 at a concentration of 4,20 , or $100 \%$ of the emitter, and a $30 \mathrm{~nm}$-thick electron transport layer (ETL) of tris(2,4,6-trimethyl-3-(pyridin-3-yl)phenyl)-borane (3TPYMB). All devices were capped with an electron-injecting cathode comprising a $0.5 \mathrm{~nm}$-thick layer of $\mathrm{LiF}$ and a 100 $\mathrm{nm}$-thick layer of Al. Electroluminescence (EL) intensity spectra collected for devices containing 20\% loading are shown in Figure 5d. Data for devices containing a neat emissive layer of 10c (see SI) show a significant red shift compared to the PL spectrum. We speculate that this emission may originate from an interfacial exciplex in this particular device. Upon doping with the UGH2 host, this effect is eliminated for the devices containing the 20\% (and $4 \%$, see SI) films.

To evaluate spectral compatibility with display applications, the coordinate system describing the color gamut developed by the International Commission on Illumination (CIE) is used. Deep blue emission is required to reproduce fully the visible color spectrum for displays as well as to achieve true white colors. For application in television displays, desirable deep blue emission has CIE coordinates of $x=0.131, y=0.046^{14 a}$ or $x=0.14, y=$ $0.08 .{ }^{14 \mathrm{~b}}$ The coordinates observed for the above devices based on 10a-c are reported in Table 1 (and graphically in the SI). All but the last (100\% 10c) emit in the deep blue.

Current density-voltage and brightness-voltage characteristics were also measured for devices based on each emitter. From these measurements, the external quantum efficiency ( $\eta_{\mathrm{EQE}}$ ) was calculated. The $\eta_{\mathrm{EQE}}$ is a direct measure of photons emitted from the device in the forward viewing direction per electron injected. Device performance for all emitter species and concentrations are shown in Table 1. The highest performance device overall was found to be 10a doped into UGH2 at 20\% with an EQE of 3\%. It is worth noting that for simple fluorescent emitters, a maximum theoretical efficiency of $\sim 5 \%$ is expected. ${ }^{15}$ Peak $\eta_{\mathrm{EQE}}$ values for all three compounds are realized for devices having an emissive layer composition of $20 \%$ of $\mathbf{1 0}$. This does not correlate directly with the PL efficiency $\eta_{\mathrm{PL}}$, which increases monotonically with dilution, likely reflecting a role played by the emitter in charge transport. The wide energy gap of UGH2 $(\mathrm{HOMO}=7.2 \mathrm{eV} ; \mathrm{LUMO}=2.8 \mathrm{eV})^{16}$ forces charge transport to occur in part via the emissive guest molecule, especially for holes. This is supported by the observed increase in turn-on voltage $\left(V_{\mathrm{TO}}\right)$ with decreasing concentration. These devices could likely be further optimized by using a charge transporting host and a lower guest concentration, capitalizing on the improved PL efficiency observed for more dilute films. 
In conclusion, we have shown that the arylalkynyl naphthalenes $\mathbf{1 0 a}-\mathbf{c}$, which differ in the electronic character of the substituents on two of their aromatic rings, can be efficiently prepared by an HDDA-enabled strategy. This involves just three chemical reactions from commercially available materials. The PL of each compound was measured in solution as well as in thin films produced by vapor deposition. Films having varying concentrations of 10 were produced by codeposition with UGH2. The PL of these films showed only a small red shift with increasing concentration, largely a reflection, we presume, of the twisted nature of the five aryl substituents that serve to enshroud the central naphthalene chromophore. Devices were constructed using 10a-c as the emissive species. Compound 10a shows the highest external quantum efficiency. Overall there was not a strong impact of the varying electronic character of the substituents $\left(\mathrm{H}\right.$ vs $\mathrm{OMe} v \mathrm{CO}_{2} \mathrm{Me}$ ) on the chromophores responsible for the absorption and emission spectra. These results suggest that other novel chromophoric structures that can be accessed by the powerful HDDA cascade approach may also be deserving of consideration for applications in various organic electronics settings.

\section{Supplementary Material}

Refer to Web version on PubMed Central for supplementary material.

\section{Acknowledgments}

This research was carried out with support from the National Institute of General Medical Sciences (GM65597, TRH) and the National Cancer Institute (CA76497) of the National Institutes of Health, U.S.A. (GM65597, TRH) and The Dow Chemical Company (RJH). NMR spectral data were obtained using instrumentation purchased with funds awarded through the NIH Shared Instrumentation Grant program (S10OD011952).

\section{REFERENCES}

1. For a summary of these methods see, e.g.: Tadross PM, Stoltz BM. Chem. Rev. 2012; 112:3550 3577. [PubMed: 22443517]

2. (a) Hoye TR, Baire B, Niu DW, Willoughby PH, Woods BP. Nature. 2012; 490:208-212. [PubMed: 23060191] (b) Baire B, Niu D, Willoughby PH, Woods BP, Hoye TR. Nat. Protoc. 2013; 8:501508. [PubMed: 23411632]

3. (a) Bradley AZ, Johnson RP. J. Am. Chem. Soc. 1997; 119:9917-9918.(b) Miyawaki K, Suzuki R, Kawano T, Ueda I. Tetrahedron Lett. 1997; 38:3943-3946.(c) Yun SY, Wang K, Lee N, Mamidipalli P, Lee D. J. Am. Chem. Soc. 2013; 135:4668-4671. [PubMed: 23477300]

4. (a) Holden C, Greaney MF. Angew. Chem., Int. Ed. 2014; 53:5746-5749.(b) Chen J, Palani V, Hoye TR. J. Am. Chem. Soc. 2016; 138:4318-4321. (and references therein). [PubMed: 27010747]

5. For a recent study of benzyne electrophilicity in which the relative reactivities of various trapping agents were exploited, see: Fine Nathel NF, Morrill LA, Mayr H, Garg NK. J. Am. Chem. Soc. 2016; 138:10402-10405. [PubMed: 27480639]

6. Wittig G, Knauss E. Chem. Ber. 1958; 91:895-907. (b) This reaction has been performed by countless numbers of students in introductory organic chemistry laboratories for decades: Fieser LF. Organic Experiments. 1964Heath and Co. Fieser LF, Haddadin MJ. Can. J. Chem. 1965; 43:1599_ 1606. (c) Reactions of TPCP with benzyne derivatives have been described in dozens of publications. For the most recent, see: Pozo I, Cobas A, Peña D, Guitián E, Pérez D. Chem. Commun. 2016; 52:5534-5537.

7. (a) Cacioppa G, Carlotti B, Elisei F, Gentili PL, Marrocchi A, Spalletti A. Phys. Chem. Chem. Phys. 2016; 18:285-294. (and references therein). [PubMed: 26607138] (b) Giguere J-B, Sariciftci N-S, Morin J-F. J. Mater. Chem. C. 2015; 3:601-606.(c) Zhang C-H, Wang L-P, Tan W-Y, Wu S-P, Liu 
X-P, Yu P-P, Huang J, Zhu X-H, Wu H-B, Zhao C-Y, Peng J, Cao Y. J. Mater. Chem. C. 2016; 4:3757-3764.(d) Broggi A, Tomasi I, Bianchi L, Marrocchi A, Vaccaro L. Chem Plus Chem. 2014; 79:486-507. (and references therein).

8. As our work was progressing, the use $\mathbf{9 a}$ and $\mathbf{9 b}$ as HDDA substrates was reported; the intermediate benzyne was efficiently trapped by various cyclic dienes (furan, cyclopentadiene, pyrrole, and thiophene derivatives) Zhang M-X, Shan W, Chen Z, Yin J, Yu G-A, Liu SH. Tetrahedron Lett. 2015; 56:6833-6838.

9. Bromination of dimethyl dipropargylmalonate (i) gives ii, which is then cross-coupled with an arylethyne (iii) of choice.

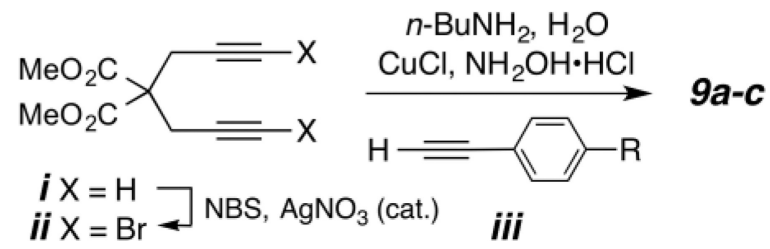

10. Mei J, Leung NLC, Kwok RTK, Lam JWY, Tang BZ. Chem. Rev. 2015; 115:11718-11940. [PubMed: 26492387]

11. Luo J, Xie Z, Lam JWY, Cheng L, Chen H, Qiu C, Kwok HS, Zhan X, Liu Y, Zhu D, Tang BZ. Chem. Commun. 2001:1740-1741.

12. (a) Scholz S, Kondakov D, Lüssem B, Leo K. Chem. Rev. 2015; 115:8449-8503. [PubMed: 26230864] (b) Reineke S, Lindner F, Schwartz G, Seidler N, Walzer K, Lüssem B, Leo K. Nature. 2009; 459:234-238. [PubMed: 19444212] (c) Sun Y, Giebink NC, Kanno H, Ma B, Thompson ME, Forrest SR. Nature. 2006; 440:908-912. [PubMed: 16612378] (d) Sun Y, Forrest SR. Appl. Phys. Lett. 2007; 91:263503.

13. (a) Chen W-C, Lee C-S, Tong Q-X. J. Mater. Chem. C. 2015; 3:10957-10963.(b) Zhu M, Yang C. Chem. Soc. Rev. 2013; 42:4963-4976. [PubMed: 23436004] (c) Chi Y, Chou P-T. Chem. Soc. Rev. 2010; 39:638-655. [PubMed: 20111785] (d) Lee M-T, Chen H-H, Liao C-H, Tsai C-H, Chen CH. Appl. Phys. Lett. 2004; 85:3301-3303.(e) Wu C-H, Chien C-H, Hsu F-M, Shih P-I, Shu C-F. J. Mater. Chem. 2009; 19:1464-1470.(f) Kim S-K, Yang B, Ma Y, Lee J-H, Park J-W. J. Mater. Chem. 2008; 18:3376-3384.

14. (a) Parameter values for ultra-high definition television systems for production and international programme exchange. Recommendation ITU-R BT.2020-2 of the International Telecommunication Union: https://www.itu.int/dms_pubrec/itu-r/rec/bt/R-REC-BT. 2020-2-201510-I!!PDF-E.pdf (accessed September 3, 2016). Farrell JE, Wandell BA. Kriss M. Image Systems Simulation. Handbook of Digital Imaging. 2015; New YorkWileyChapter 8

15. (a) Uoyama H, Goushi K, Shizu K, Nomura H, Adachi C. Nature. 2012; 492:234-238. [PubMed: 23235877] (b) Kim KH, Ma JY, Moon CK, Lee JH, Baek JY, Kim YH, Kim JJ. Adv. Opt. Mater. 2015; 3:1191-1196.

16. Ren X, Li J, Holmes RJ, Djurovich PI, Forrest SR, Thompson ME. Chem. Mater. 2004; 16:47434747. 
a
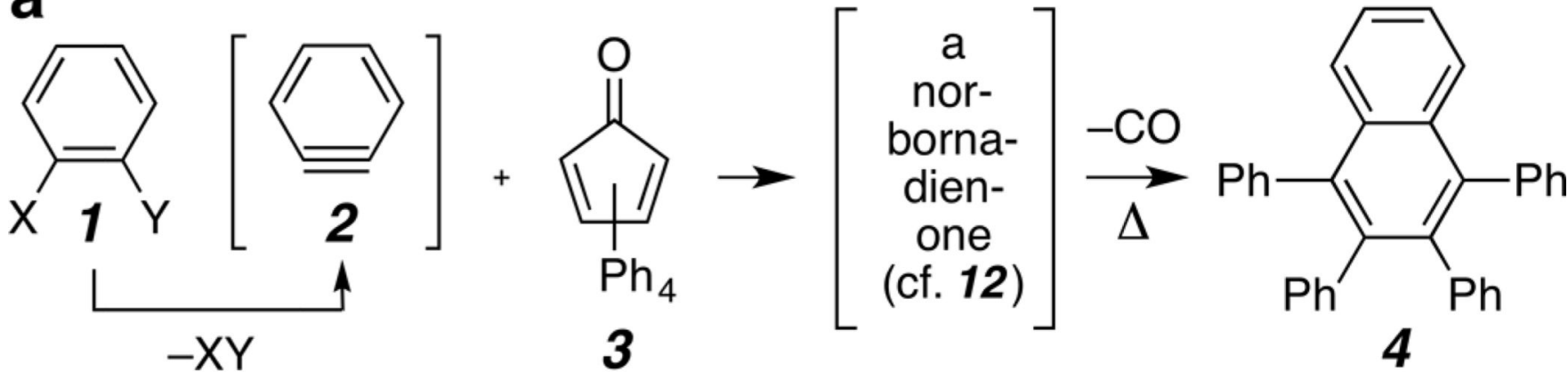

b
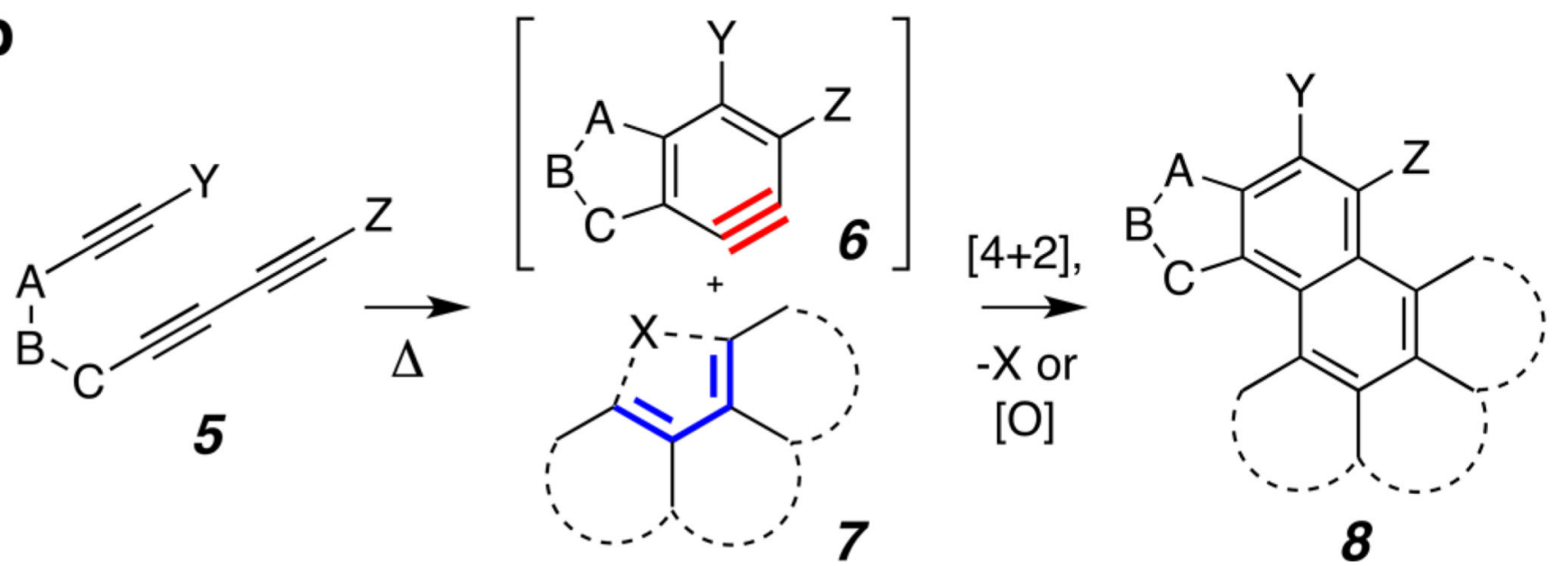

Figure 1.

(a) Traditional benzyne generation ${ }^{1}$ involves removal of a pair of ortho substituents from a benzenoid precursor 1; Diels-Alder reaction of $\mathbf{2}$ with tetraphenylcyclopentadienone (3) produces naphthalene derivatives, following thermal extrusion of carbon monoxide. (b) The HDDA thermal cycloisomerization ${ }^{2,3}$ (5 to 6) produces benzynes that are more complex and diverse in structure; trapping with myriad dienes like $\mathbf{7}$ is envisioned to produce novel highly conjugated adducts like $\mathbf{8}$. 


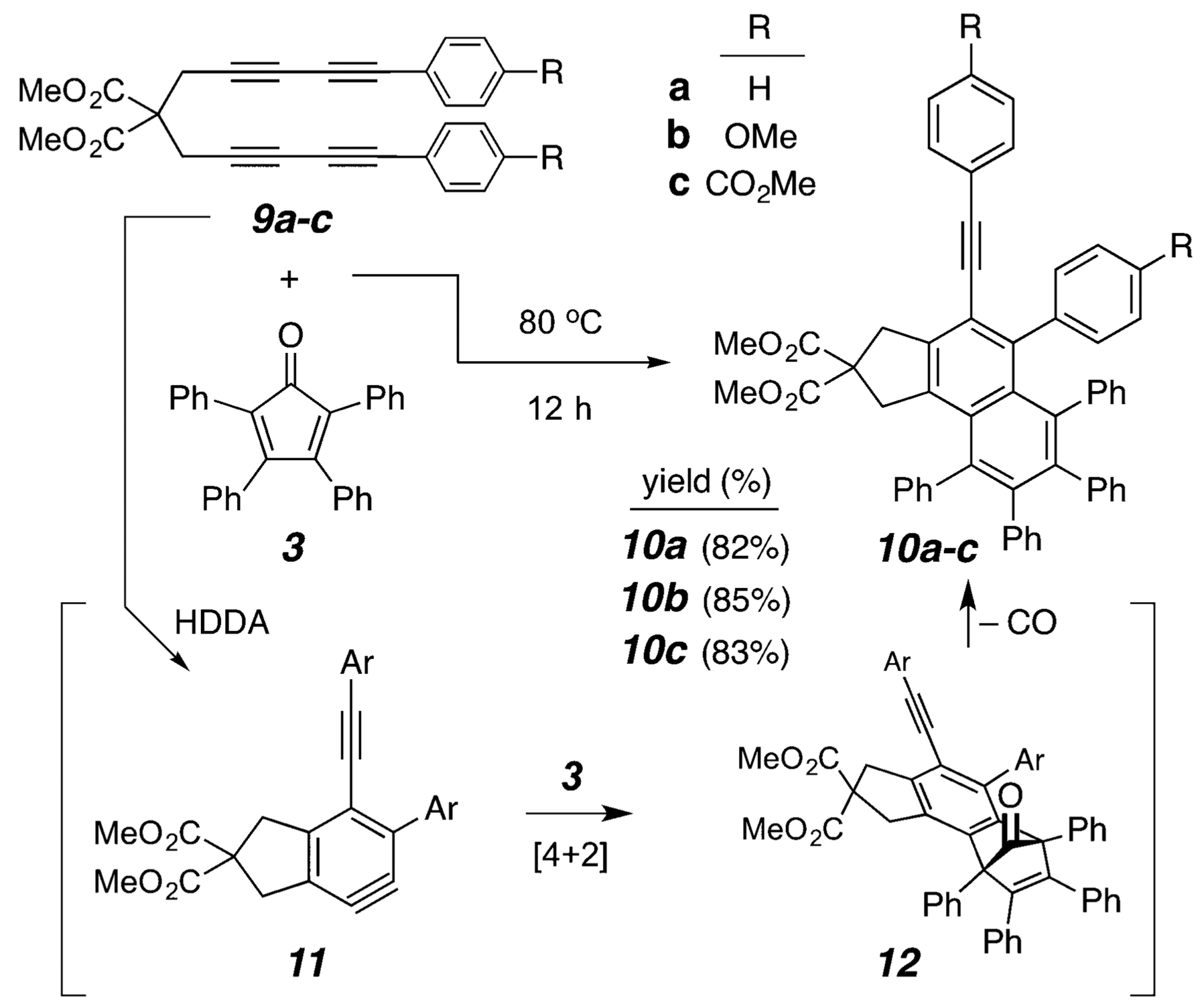

Figure 2.

HDDA cascade reaction between tetraynes $\mathbf{9}$ and $\mathbf{3}$, proceeding via benzynes $\mathbf{1 1}$ and their Diels-Alder adducts 12, which ejects carbon monoxide to produce the fluorophores $\mathbf{1 0}$ in excellent yield. 
a

\section{b}

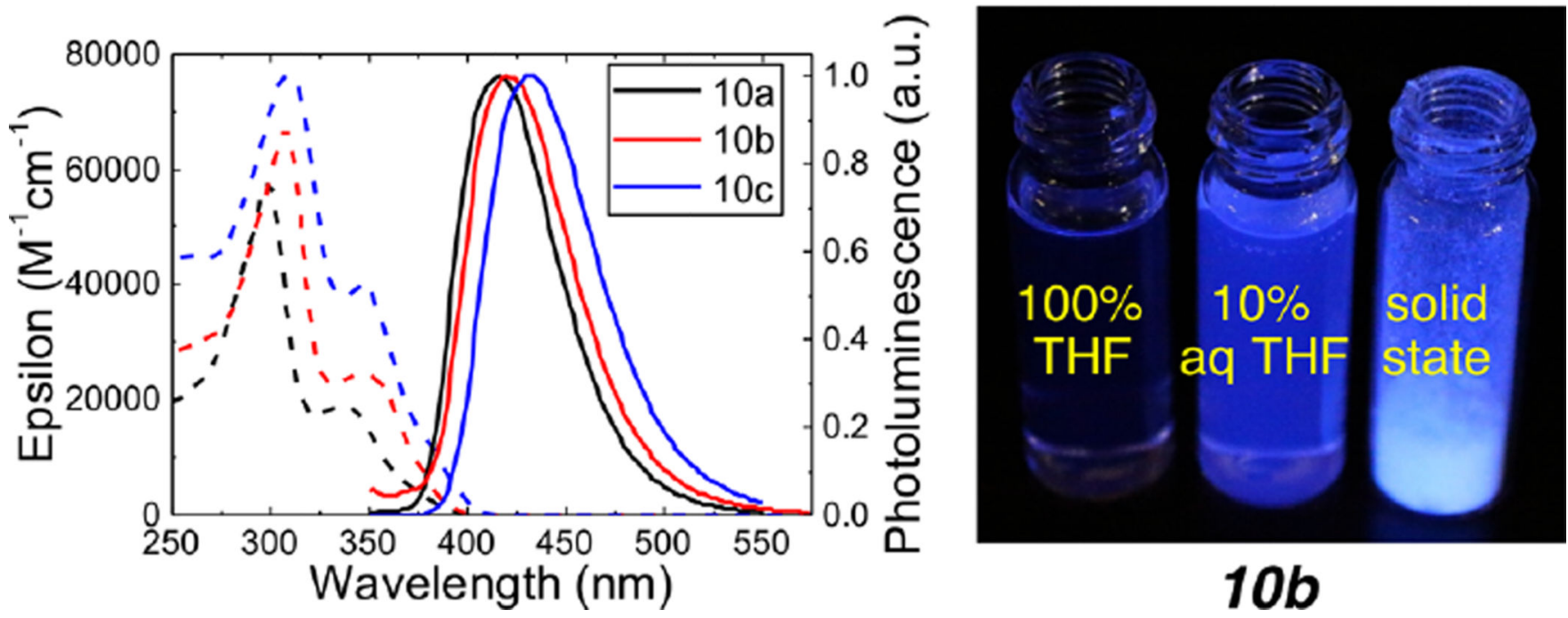

Figure 3.

(a) Solution absorption (at $10^{-5} \mathrm{M}$, dashed lines) and normalized photoluminescence (PL) emission (at $10^{-6} \mathrm{M}$, solid lines) spectra in THF. (b) Emission seen from 10b in various media/states. 
a

\section{b}
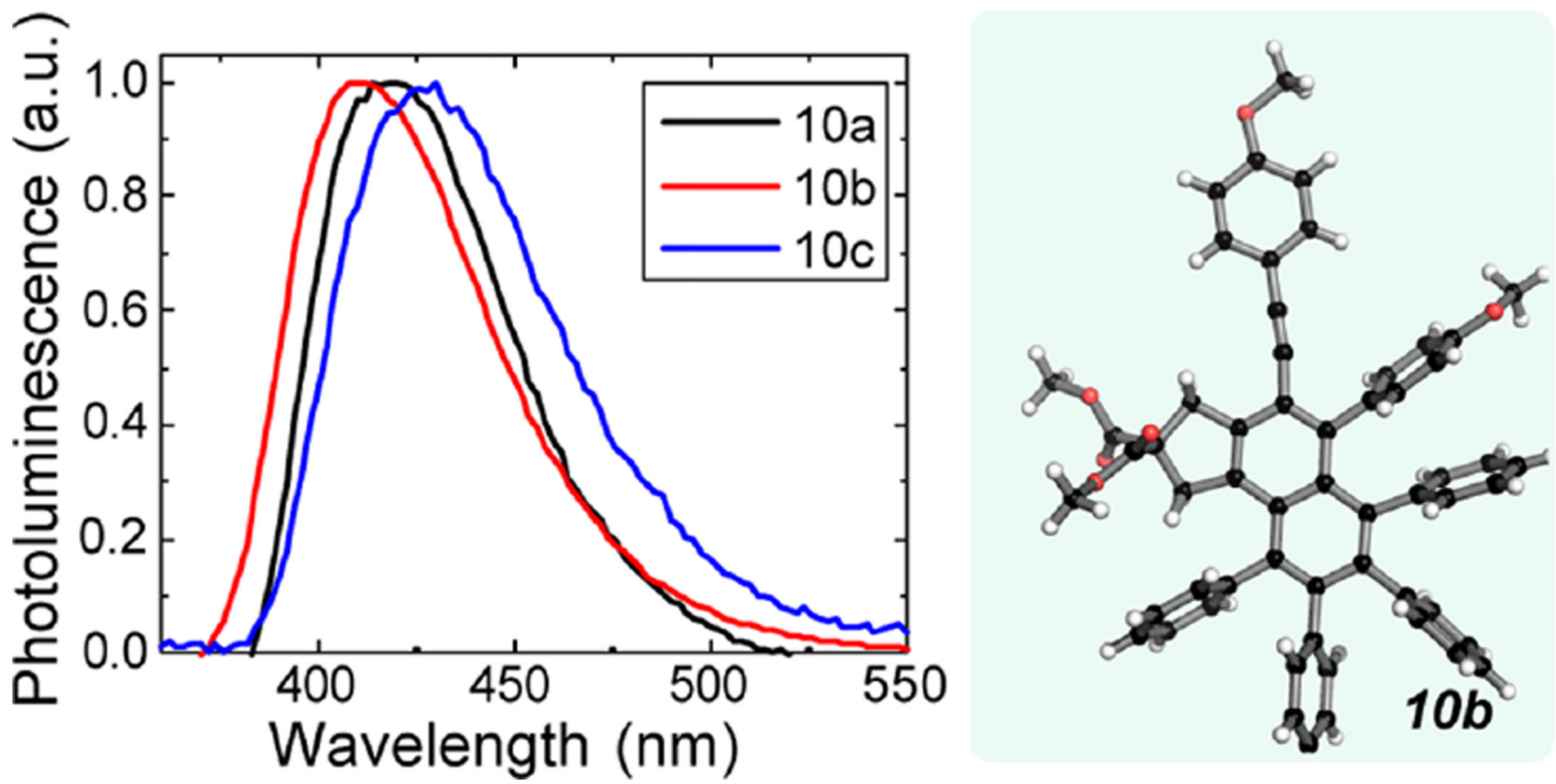

Figure 4.

(a) Thin film (20 nm) normalized PL spectra of 20:80 mixtures of 10a-c codeposited with the wide-gap host UGH2 under excitation at a wavelength of $300 \mathrm{~nm}$. (b) Single crystal Xray structure of $\mathbf{1 0 b}$ showing the relative orientation in the solid state of all aryl substituents with respect to the core naphthalene ring. 

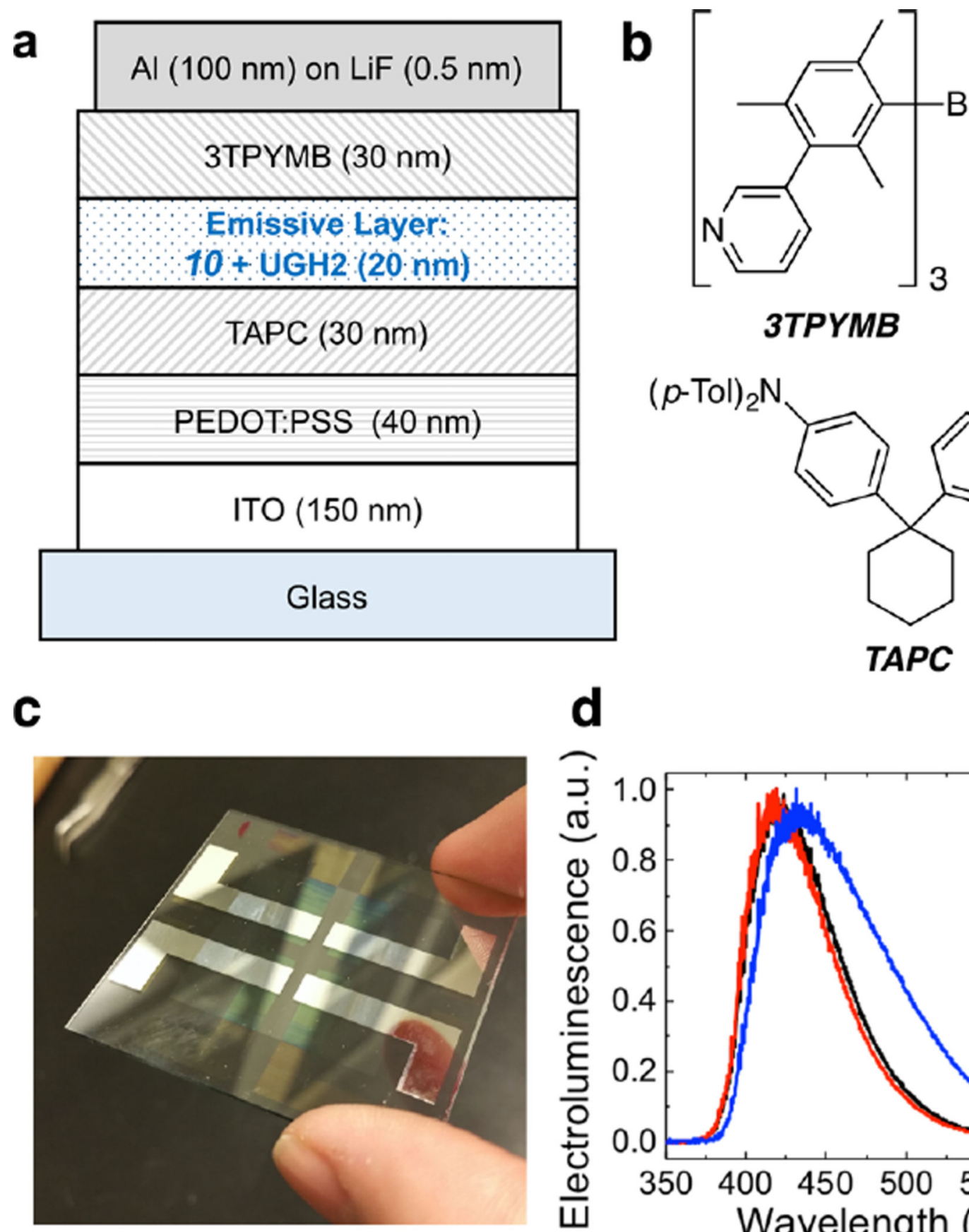<smiles>[SnH3]c1ccccc1</smiles>

UGH2

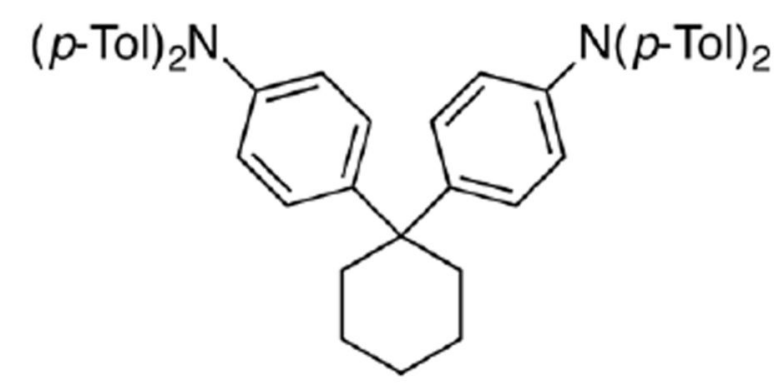

TAPC

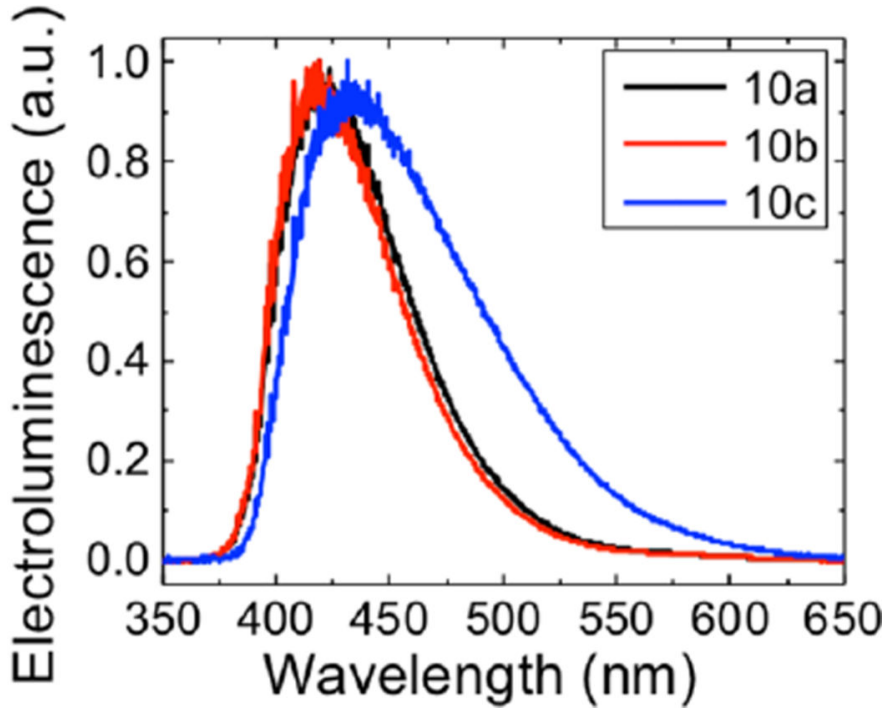

Figure 5.

OLEDs (a) Device architecture. (b) Compounds used as the electron transport layer (3TPYMB), the host in the emissive layer (UGH2), and the hole transport layer (TAPC). (c) Photograph of a typical device. (d) Electroluminescence intensity (normalized) from devices using 20 wt $\%$ of emitter 10 doped in UGH2. 


\section{롤 \\ 일}

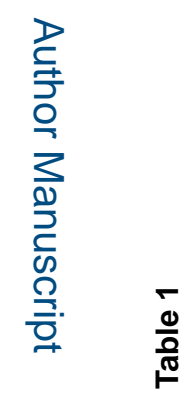

롤

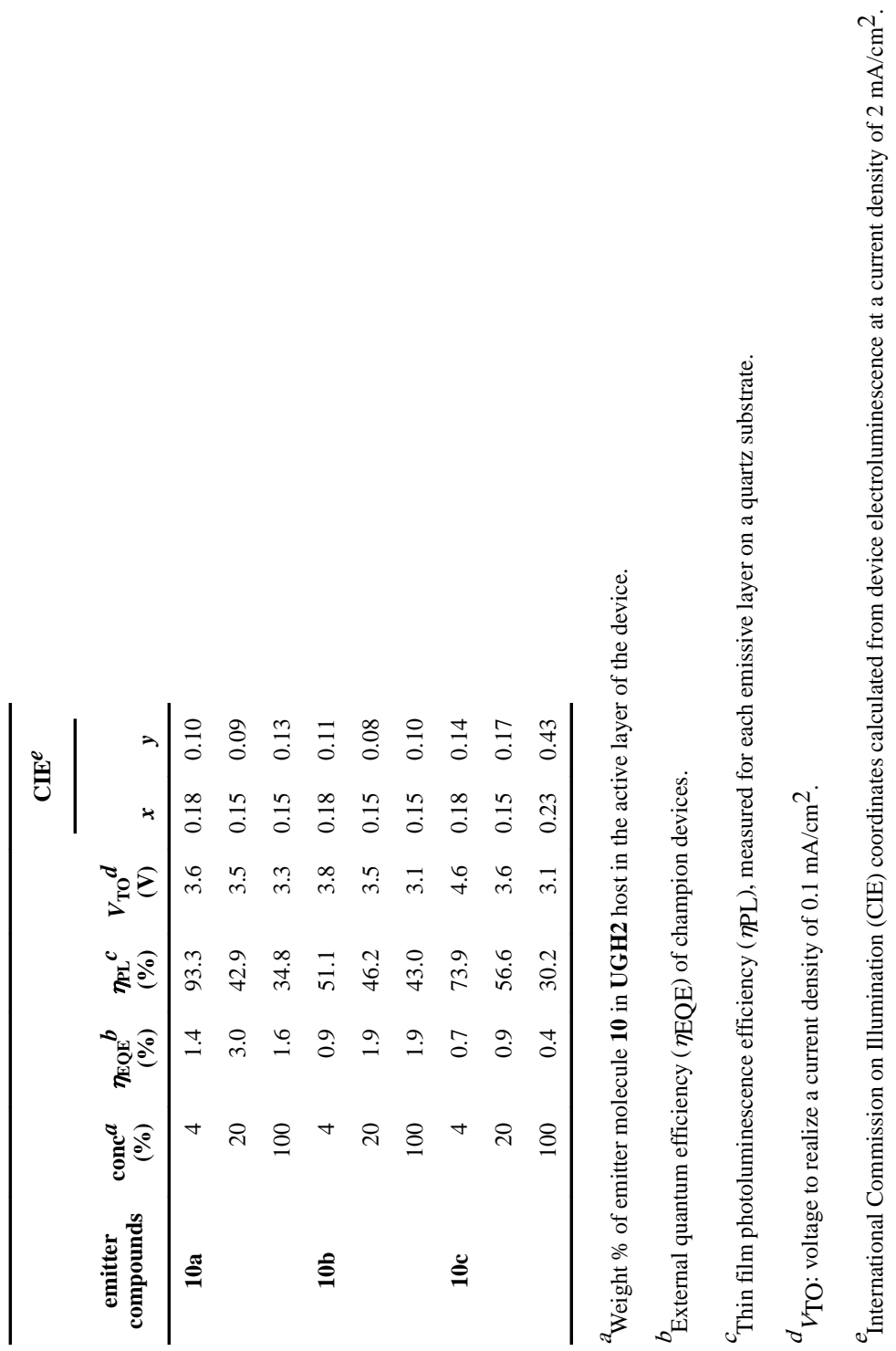

J Am Chem Soc. Author manuscript; available in PMC 2017 October 05. 